\title{
Loglet SIFT for Part Description in Deformable Part Models: Application to Face Alignment
}

Qiang Zhang

q.zhang.13@warwick.ac.uk

Abhir Bhalerao

abhir.bhalerao@warwick.ac.uk

\author{
Department of Computer Science \\ University of Warwick \\ Coventry, UK
}

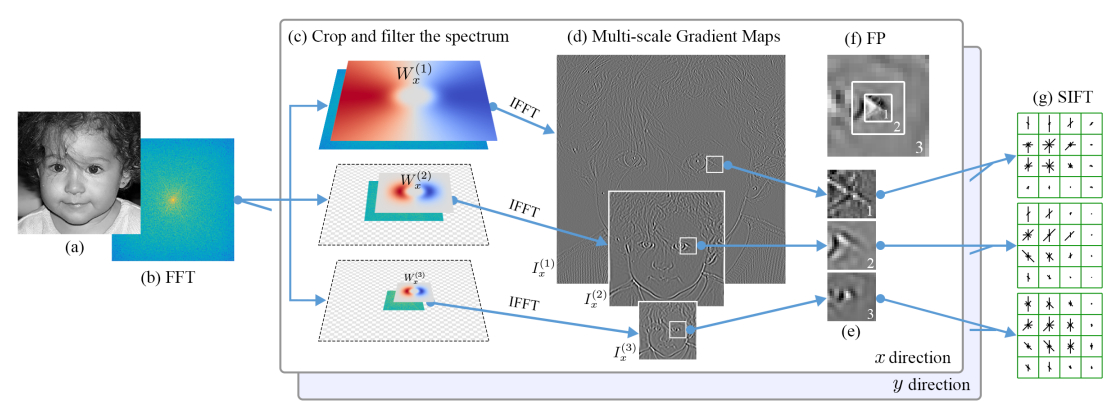

Figure 1: Overview of extracting a loglet-SIFT part descriptor. (a) An image example. (b) The Fourier spectrum of the image. (c) Filter and crop the spectrum with the filter banks. The process with $x$ direction is shown as an example. (d) Pyramids of the gradient maps representing multi-scale structures are obtained directly from the filtered spectrum. (e) At a landmark, patches with the same size in pixels are extracted as a multi-scale local descriptor. (f) The patches represent coherent scales and domain sizes forming a feature pyramid. (g) The Loglet-SIFT descriptor is obtained like SIFT, by accumulating directional gradients from each of the gradient patches.

Deformable Part Model (DPM) have emerged as the leading approach for accurate landmark detection in applications such as face alignment. A DPM describes an object by local parts and the spacial relationships of the parts. Part descriptors seek a representation of local structures which preserves intrinsic properties and discriminative information, while exhibiting invariance to changes such as illumination, scale and variations in appearance across instances. We propose a new local feature descriptor called loglet-SIFT, which enhances a number of invariances, i.e., the invariance to illumination by the local pooling of SIFT and the suppression of slow varying mean level by the wavelets, as well as the invariances to noise by SIFT, and to sample shift by loglets. An overview of the proposed method can be found in Fig 1 .
We integrate our descriptor into a DPM driven by a supervised descent method and validate its performance in the face alignment scenario. We compare the performance of our Fourier domain designed filters with spatially designed gradient filters, and compare our descriptor with conventional SIFT. We further present the comparison against several state-ofthe-art methods on two popular datasets: HELEN and 300-W. Experimental results show that the new descriptor improves the performance of the DPM by a significant margin. We achieve state-of-the-art performance on HELEN and 300-W common dataset, and comparable performance on the $300-\mathrm{W}$ challenging dataset. The proposed descriptor can be readily integrated in other gradient and SIFT based deformable part models. 